\title{
Disease knowledge and patient education are key players for a better quality of life in vascular surgery patients
}

\author{
Andrej Udelnow · Verena Hecht · Ivo Buschmann · Catharina Wilbrandt · Udo Barth · Frank Meyer · Zuhir Halloul
}

Received: 10 November 2020 / Accepted: 13 November 2020 / Published online: 4 December 2020

(c) The Author(s) 2020

\begin{abstract}
Summary
Background The aim of this prospective investigation was to determine factors that influence the verbal disease knowledge level of the patient after the informed consent (IC) talk and to assess whether it can be improved by additional education. Furthermore, the factors underlying quality of life (QoL) after treatment, among them knowledge, education, and ambulatory care, were investigated.

Methods Consecutive patients who had undergone vascular surgical intervention for abdominal aortic aneurysm (AAA), thrombendarteriectomy (TEA) of the femoral bifurcation, or digital subtraction angiography (DSA) with or without endovascular intervention for arterial occlusive disease (AOD) were included over a defined study time period. They were divided randomly into (i) standard IC talk, (ii) extended IC talk with a PowerPoint lecture (PP; Microsoft Inc.,
\end{abstract}

PD Dr. med. habil. A. Udelnow · Dr. med. V. Hecht Brandenburg Municipal Hospital, Department of Vascular and Endovascular Surgery/Phlebology, Brandenburg Medical School “Theodor Fontane”, Brandenburg, Germany

Prof. Dr. med. habil. I. Buschmann Brandenburg Municipal Hospital, Department of Angiology, Brandenburg Medical School “Theodor Fontane”, Brandenburg, Germany

\section{Dr. med. C. Wilbrandt}

Department of Anesthesiology and Intensive Care, Otto-von-Guericke University of Magdeburg with University Hospital, Magdeburg, Germany

Dr. med. U. Barth · Prof. Dr. med. habil. F. Meyer, MBA, M.Sc. · Prof. Dr. med. habil. Z. Halloul $(\bowtie)$

Division of Vascular Surgery, Department of General, Abdominal, Vascular and Transplantation Surgery, Otto-von-Guericke University of Magdeburg with University Hospital, Leipziger Str. 44, 39120 Magdeburg, Germany zuhir.halloul@med.ovgu.de
Redmond, WA, USA), and (iii) extended IC talk with a walking diary (WD). The patients filled out questionnaires before and after treatment and a RAND36 Health Survey during follow-up. The disease knowledge level was quantified by six verbal items. Group comparisons were performed by matched-pair analysis (MPA) and factor analysis by multiple regression analysis and multivariate analysis of variance (MANOVA).

Results From January 2015 until November 2016, $n=198$ patients were enrolled. Remembrance and comprehension rates were in general low and did not improve significantly with additional PP or WD. Age was inversely associated with disease knowledge. Age was also inversely and WD/PP and knowledge level directly associated with mid-term QoL. However, other factors such as care provision and social status were important as well.

Conclusion Communication with and education of vascular surgery patients is one of the mainstays of treatment and should be continued beyond the legal and formal requirements during treatment and after discharge from hospital in order to keep the QoL as high as possible.

Keywords Informed consent - Comprehension · Physician-patient interaction · Prospective investigation

\section{Introduction}

The patient's informed consent (IC) is a legal requirement for all medical interventions. Recent studies have shown that signing the IC form does not necessarily mean comprehension of the disease and the planned therapy or remembrance of the given information [1]. 
The aim of the present study was to detect the factors underlying patients' ability or inability to recall the most important information on the planned treatment. Furthermore, whether an extended IC talk may improve patients' comprehension and treatment satisfaction compared to a standard IC talk for peripheral arterial obstructive disease (PAOD) was investigated. The working hypotheses was that interactive education using PowerPoint presentations (PP) supports the comprehension of the IC talk and that self-experience in a walking diary (WD) would guide the patient to a more realistic expectation concerning the walking ability after treatment and thereby to better compliance. Disease knowledge, compliance, and lifestyle changes are intercorrelated [2]. Since healthrelated quality of life (QoL) is also lower in PAOD patients in a manner comparable to other cardiovascular diseases [3], the associations between disease knowledge, lifestyle education, and QoL were further analyzed. PAOD patients treated by digital subtraction angiography (DSA) with or without intervention or by thrombendarteriectomy of the femoral bifurcation (TEA) were included. These groups were comparable in terms of their main diagnosis (PAOD) but differed in terms of the invasiveness and potential risks of treatment and, therefore, in terms of the information they received about the planned treatment. Moreover, patients treated electively for non-ruptured abdominal aortic aneurysm (AAA) by endovascular or open surgical means were included as a third group, which differed from the first ones in that treatment was entirely prophylactic (aiming at prevention of aneurysm rupture, not primarily at improvement of QoL) and in that most patients did not suffer from progressed PAOD.

\section{Patients and methods}

\section{Study design}

A prospective observational study including consecutive patients hospitalized for

1. elective endovascular or open surgical non-ruptured AAA,

2. treatment of PAOD by DSA with or without percutaneous transluminal angioplasty (PTA)/stenting, (or)

3. TEA of the femoral bifurcation

was conducted from January 2015 until November 2016 at the Department of General, Visceral, Vascular, and Transplantation Surgery of the Magdeburg University. Exclusion criteria were inability to give informed consent, decline of treatment or study participation, and ambulant or emergency surgery.
Standardized IC talk, interventions, and questionnaires

Participants were assigned to standard IC talk (standard group) between January 2015 and August 2016 (excluding February 2016), a one-hour long extended IC talk using an additional PowerPoint presentation in February 2016 (PP group), or a walking diary between September 2016 and November 2016 (WD group). When one of these treatments was indicated, the patients were explained the diagnosis and the recommended and alternative treatment strategies by a vascular surgeon at the ambulatory consultation and were provided with the corresponding German standard IC forms (Thieme proCompliance $\mathrm{GmbH}$, Erlangen, Germany) for self-information at home. The standardized IC talk was then performed about 1 week before hospital admission using this form. They were further asked about their willingness to participate in the study. In case of consent, they were included into the present investigation after assessing the absence of exclusion criteria and signing the IC form. After the standardized IC talk (prepared as PowerPoint [Microsoft Inc., Redmond, WA, USA] presentation about AAA, TEA, or DSA and explained to the appropriate patients), a one-hour long patient education was performed in the talking group. The patients had the opportunity to ask for more details and explanation concerning the diagnosis, lifestyle changes, and the techniques and possible complications of the planned procedure. After the indication for surgery, the walking group received flyers about their disease and the importance of walking training and a walking diary plus pedometer. The patients were instructed to note down their steps every day according to the pedometer until the follow-up care. On the day of hospital admission, as shown in Fig. 1, the patients received a first questionnaire on the day of admission, before the planned procedure, containing 20 questions and evaluating the knowledge about the disease, self-information, smoking habits, and treatment expectations. In order to quantify the knowledge level, six items were asked and correct answers summed up: diagnosis, planned treatment, incision site, and three possible complications during or after the intervention. The answers were considered correct if the patients verbalized a synonymous word (i.e., "vessel dilatation" instead of "aneurysm") and were considered incorrect if the patient left the field empty or filled in something completely different (i.e., "hypertension" instead of "peripheral atherosclerosis"). After surgery and before discharge, they completed a second questionnaire containing 8 items evaluating treatment satisfaction. During follow-up, within 3 up to 6 months, they filled out a modified standardized quality of life questionnaire (RAND 36Item Health Survey [version 1.0]) [4] combined with parts of the World Health Organization Quality of Life questionnaire [5]. 
Fig. 1 Flowchart of the study. DSA Digital Substraction Angiography, PTA Percutaneous Thrombarterioplasty

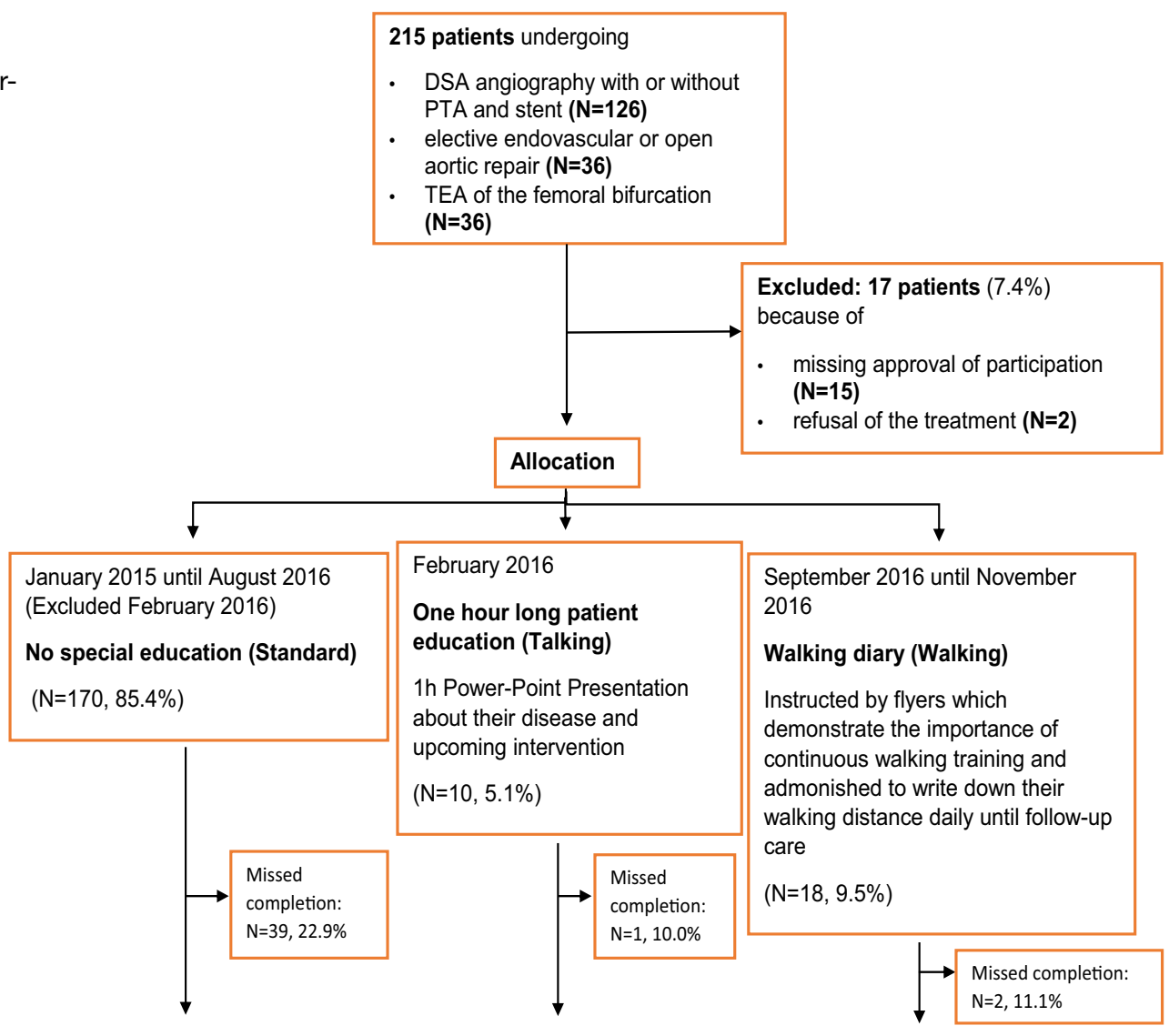

After informed consent talk and before surgery $(\mathrm{N}=156)$ :

Questionnaire evaluating the knowledge about the disease and treatment expectations

Missed completion: $\mathrm{N}=41$

After surgery and before discharge $(\mathrm{N}=\mathbf{1 1 5})$

Questionnaire evaluating the treatment satisfaction

Missed completion: $\mathrm{N}=54$

At the time of out-patient follow-up care $(\mathrm{N}=61)$

Standardized Quality of Life Questionnaire (SF-36)

\section{Ethics}

This study was approved by the Otto-von-Guericke University ethics board and registered at the German Register of Clinical Trials (study No. DRKS00014050). The patient data were pseudonymized and stored according to the legal requirements of the German district Saxony-Anhalt. The decision of the patient to take part in the investigation or not did not influence any decision regarding care, diagnostics, and treatment. Both the study participation consent form and the questionnaires were blinded to the health care providers. All procedures involving human participants were in accordance with the ethical standards of the institutional and/or national research committee and with the 1964 Helsinki declaration and its later amendments for biomedical research or comparable ethical standards.

\section{Statistics and software}

The participants were asked in the first questionnaire to write down a keyword they remembered from the IC talk regarding the items incision site, their diagnosis, their planned treatment, and three possible complications of the treatments. Synonymous words were also counted as correct, i.e., atherosclerosis instead of PAOD. The number of correctly remembered key- 


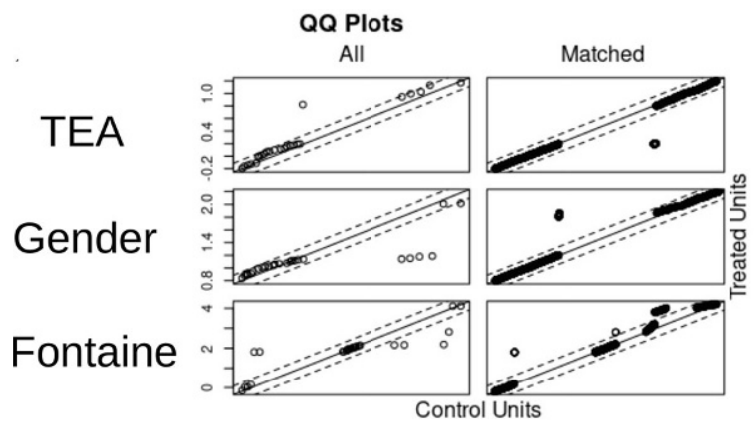

QQ Plots

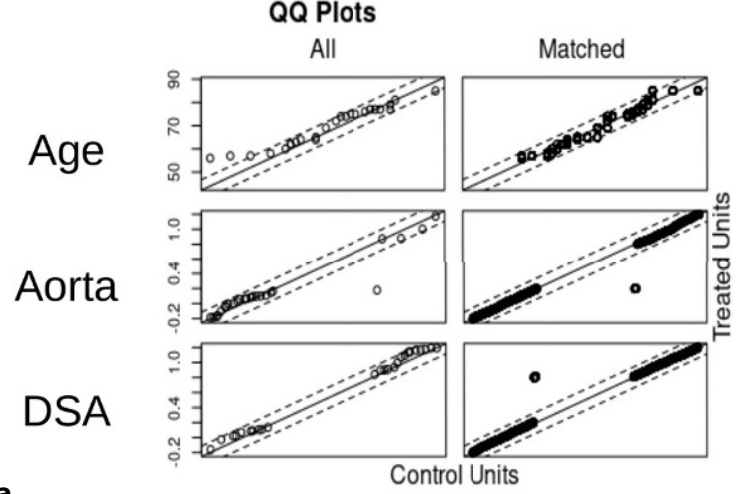

$a$

Fig. 2 Group comparison results of the first questionnaire (comprehension and remembrance). Patients with either Power-Point presentation in addition to the IC talk or walking diary were taken together as "extended IC talk" and compared with the standard IC talk patients: a QQ-plots of the group matching showing improved balance of the covariates

words were summarized in the variable "knowledge," which ranged from zero (did not remember any item) up to six (remembered all items including three possible complications).

Comparisons between groups were made using the Fisher's exact test for binary variables and independent $t$-test for continuous variables if normally distributed, and the U test by Mann and Whitney if not. A $p$-value less than 0.05 was accepted as significant. Multiple linear regression analysis (stepwise backward algorithm) was used to identify independent predictors of the variable knowledge. Statistical analysis was conducted using $\mathrm{R}$ (R Foundation for Statistical Computing, Vienna, Austria) [6]. The following packages were used for the corresponding statistical methods: MatchIt [7] for matched-pair analysis using the genetic algorithm, multivariate analysis of variance (MANOVA) using mvnormtest [8] for the test on multivariate normality, and the manova function of the standard software.
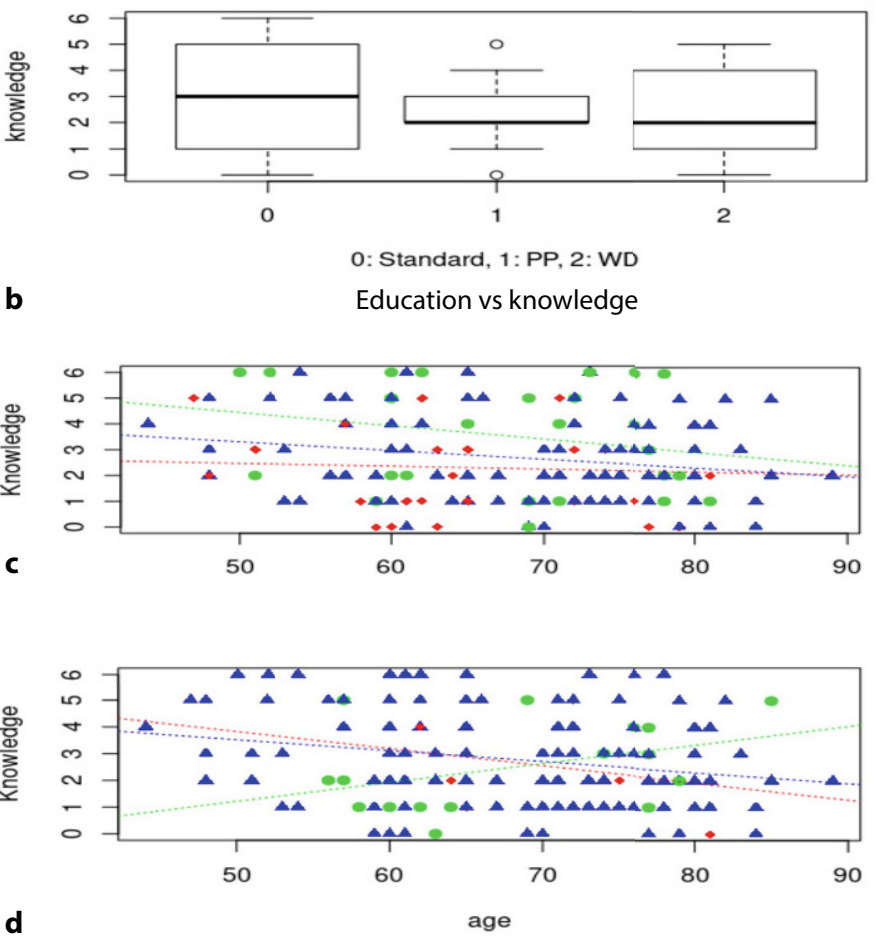

between groups. b Box plots comparing the knowledge levels in the matched standard, PP and WD subgroups (no significant difference). c, d Scatterplots of the relationships between age and knowledge within different subgroups: $\mathbf{c}$ treatment subgroups, d IC talk subgroups, containing a linear regression line for each subgroup

\section{Results}

\section{Comparison of disease knowledge and comprehension resulting from the IC talk between (extended) IC talk groups}

From January 2015 until November 2016, $n=215$ patients corresponded to the inclusion criteria and 198 patients were enrolled and subdivided into groups as shown in Fig. 1. The first questionnaire was filled out by $n=156$ patients, without missing values (MV) by $n=148$ patients. One of the aims of the study was to compare the knowledge levels between the standard, PP, and WD groups. Since patient characteristics may differ between the groups and may cause confounding bias, a matched-pair analysis was conducted as demonstrated by the QQ plots in Fig. 2a, where the standard group was matched by the two extended IC talk groups taken together. Fig. $2 \mathrm{~b}$ shows the boxplots of the mean and median knowledge levels in the three subgroups, which did not differ relevantly. A one-way analysis of variance (ANOVA) was performed and significant differences could neither be calculated by the linear model ( $p: 0.584$, aov function of the R software) nor by the post-hoc analysis (PP and WD vs. standard group, $p: 0.884$ and $p: 0.877$, respectively, and PP vs. 
Table 1 Multivariate linear regression of predicting factors for disease knowledge, first and last step

\begin{tabular}{|c|c|c|}
\hline & Estimate & $p$-value \\
\hline \multicolumn{3}{|l|}{ First step (initial model) } \\
\hline Intercept & -2.367 & $<0.0001$ \\
\hline Gender & 0.016 & 0.92 \\
\hline Duration of the informed consent talk & 0.003 & 0.48 \\
\hline $\begin{array}{l}\text { Duration between informed consent talk and } \\
\text { filling up the first questionnaire }\end{array}$ & -0.004 & 0.54 \\
\hline Age & -0.016 & $0.017^{\star}$ \\
\hline DSA & -0.291 & 0.12 \\
\hline TEA & -0.410 & $0.042^{*}$ \\
\hline Fontaine classification & 0.023 & 0.69 \\
\hline Self-Information before IC talk & 0.273 & 0.056 \\
\hline Chance to ask questions before IC talk & 0.057 & 0.65 \\
\hline Estimated quality of life after intervention & -0.183 & 0.15 \\
\hline Concerned about the risks & -0.026 & 0.77 \\
\hline Questions were helpfully answered & 0.075 & 0.54 \\
\hline Smoking & -0.118 & 0.25 \\
\hline Diabetes mellitus & 0.226 & 0.082 \\
\hline Coronary heart disease & 0.153 & 0.24 \\
\hline Atrial fibrillation & -0.096 & 0.61 \\
\hline Extended IC talk (PP or WD) & -0.026 & 0.78 \\
\hline \multicolumn{3}{|l|}{ Last step (containing only significant terms) } \\
\hline Intercept & 1.92 & $<0.001^{*}$ \\
\hline Age & -0.013 & $0.010^{*}$ \\
\hline DSA & -0.248 & $0.047^{*}$ \\
\hline TEA & -0432 & $0.007^{\star}$ \\
\hline Self-information before IC talk & 0.283 & $0.031^{*}$ \\
\hline
\end{tabular}

$\mathrm{WD}, p: 0.997)$. Furthermore, we performed a linear regression analysis in order to determine the cofactors associated with the knowledge level after the IC talk, as listed in Table 1. Age and the PAOD-associated interventions DSA and TEA contributed to lower and the statement of the patient that she/he has read the IC talk form already before the IC talk to higher knowledge level. However, as shown in Fig. 2c, d, the dependencies of the knowledge level on age are not unequivocally observable in the different subgroups since knowledge did not decrease with age in patients operated on for AAA (Fig. 2c), and even increased with age in the WD subcohort (Fig. 2d).

Summarizing, we can state that an extended IC talk via PP or WD did not significantly improve the disease knowledge. Determining factors were age, PAOD (TEA and DSA) compared to AAA, and self-information by reading the IC form prior to the IC talk.

\section{Satisfaction with staff and treatment}

The knowledge level of the patients and its contributing factors may also affect the treatment satisfaction and the compliance of patients. The second questionnaire, filled out on the day of discharge, contained questions about the satisfaction with the staff and the treatment. Because the number of completed questionnaires was lower than that of the first questionnaire, a new matched-pair analysis was performed. The QQ plots are outlined in Fig. 3a. The patients were asked to score the staff by grades from 1 (very good) up to 5 (insufficient). Fig. 3b-e show the comparisons of the mean scores between the standard and extended IC talk groups by box-whisker plots for the treatment (b, $p: 0.61$ ), the surgeons (c, $p: 0.89$ ), the nursing staff (3D, $p: 0.78$ ), and the general condition (e; by the scoring 0 : feeling worse than before treatment; 1 : feeling equally; 2 : feeling better; $p: 0.65)$, without significant differences. An extended IC talk did not relevantly improve the short-term satisfaction. However, most patients evaluated treatment, situation, and staff as "good" or "very good." Therefore, it was not possible to draw any conclusions on the relationships between education and treatment satisfaction.

\section{Long-term impact of disease knowledge and patient education on QoL}

Since treatment expectations and long-term satisfaction with treatment may also depend on the knowledge level about the disease, the mid-term QoL was assessed.

The RAND36 questionnaire was completed by $n=63$ patients (29\%) during the ambulatory interval of 6-12 months after treatment, among them $n=7$ in the extended IC talk groups. A new matchedpair analysis was performed, the QQ plots of which are shown in Fig. 4a. The mean scores of the eight domains of the RAND 36 Health Survey 1.0, which reached from zero (low QoL) up to 100 (high QoL), are outlined in Fig. $4 \mathrm{~b}-\mathrm{i}$. The mean scores were higher in the extended IC talk group in each of the domains. However, significance was reached only in the domain "role limitation: physical health" (12 vs. $65 ; p: 0.022$ ), and a trend toward a higher mean value was observed in the education group for the domain "physical functioning" (42 vs. $69, p$ : 0.07). A discriminant analysis was conducted, which determined the reciprocal relationships between the QoL domains and the grouping variable, thereby taking the cross-correlation between the domains into account (Fig. 4k). Table 2 lists the LD coefficients. The slightly overlapping histograms and the positive and negative coefficients indicate that a higher score did not unequivocally implicate membership in the extended IC talk group for all domains (starting from an a priori probability of $50 \%$ ). It seems that other factors influenced the QoL as well. 


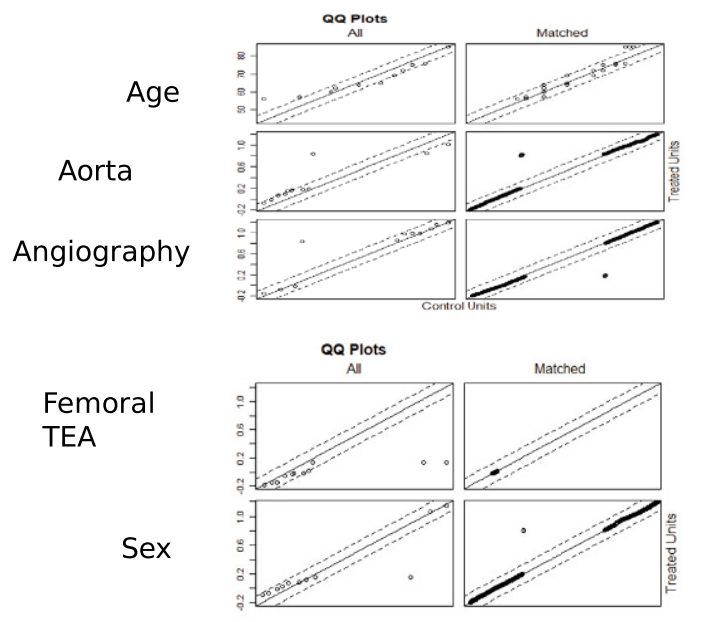

a

Fig. 3 Group comparison results of the second questionnaire (treatment satisfaction). Grouping as in Fig. 1. Scoring was possible from 1 (excellent) up to 5 (insufficient): a QQplots of the group-matching showing improved balance of the

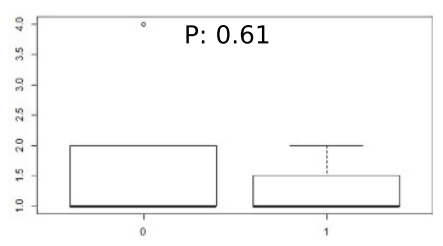

b Extended IC talk

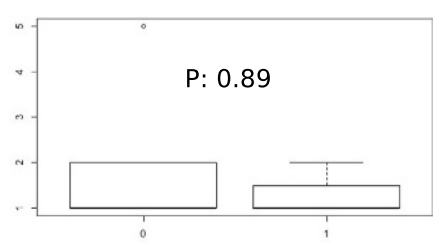

Extended IC talk

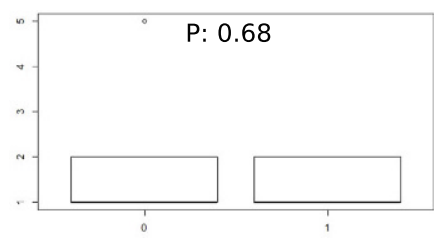

d

Extended IC talk

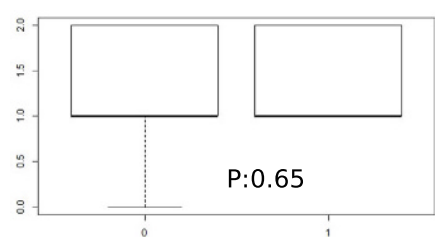

e $\quad$ Extended IC talk

covariates between groups. b-e Box plots comparing the satisfaction with b treatment, c Surgeons, d nursing staff, e general condition after treatment (Scoring from 0..2). No significant differences between the groups
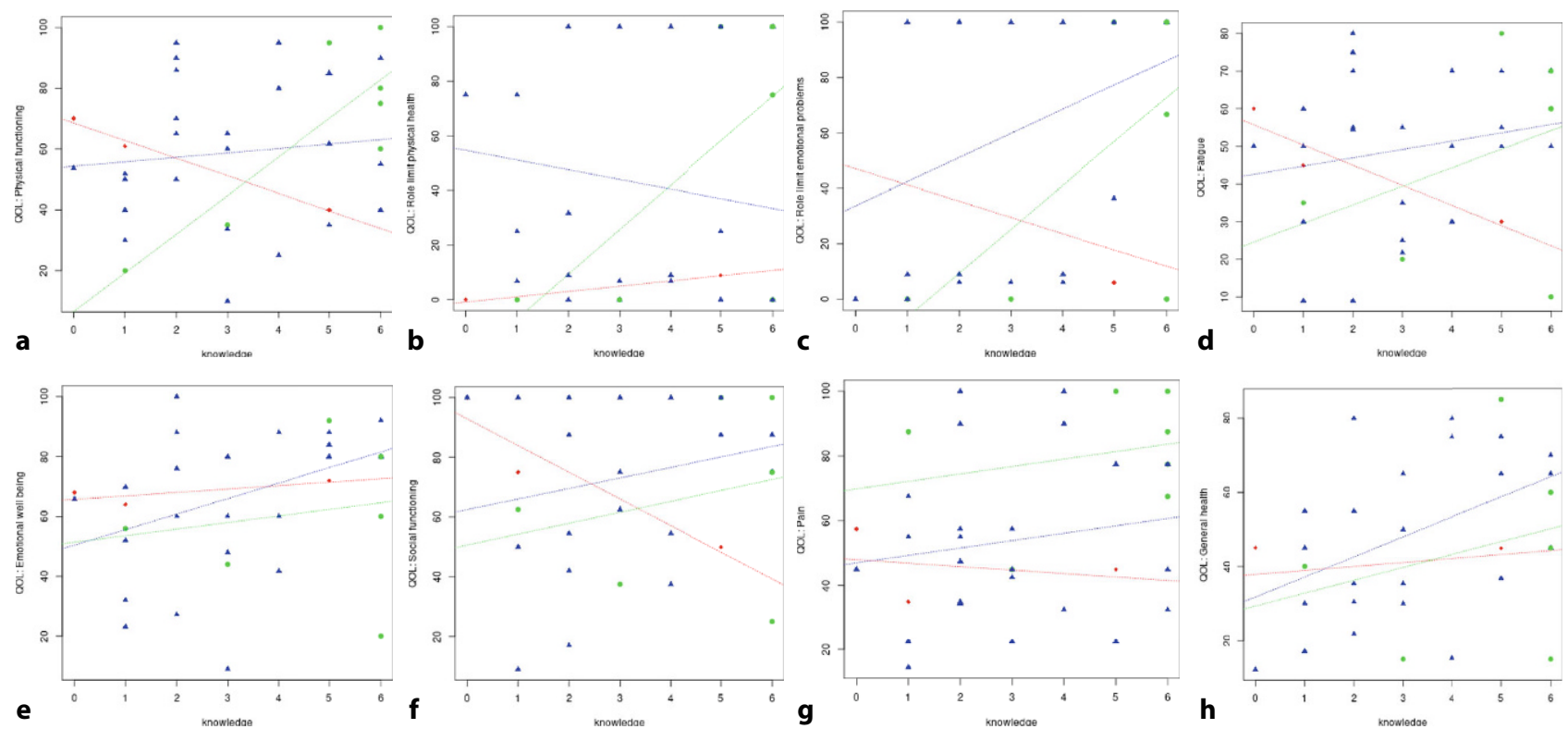

Fig. 4 Scatterplots of the knowledge vs. QoL-score relationships in Fontaine I and II subcohort for different treatment subgroups and different RAND-36 domains: a physical function-

Fig. 4 shows the scatterplots of the QoL vs. knowledge level for the RAND-36 domains in the Fontaine stage I and II subcohorts subdivided into the treatment groups, demonstrating the statistical associations between knowledge and QoL. There were positive associations between QoL and knowledge in AAA patients for each domain. The same was true in the DSA subcohort, except for the "role limit: physical health" domain, where it was negative (Fig. 4b). The TEA subgroup contained only a few cases which had completed the third questionnaire, and therefore was

ing, b role limit: physical health, c role limit: emotional problems, $\mathbf{d}$ fatigue, e) emotional well-being, $\mathbf{f}$ social functioning, $\mathbf{g}$ pain, $\mathbf{h}$ general health

not conclusive. Considering the inter- and cross-correlations between the cofactors, which influenced the QoL during the disease course, a MANOVA was performed including the eight QoL domains as response variables and the cofactors listed in Table 3 as explanatory variables. There were no significant associations in the multivariate analysis. However, the followup ANOVA revealed that belonging to the extended IC talk groups (PP or WD) was associated with the physical functioning (PF) and the social functioning (SF) domains. The Fontaine stage showed an impact on 
Table 2 LD1 coefficients resulting from discriminant analysis. Positive values indicate a positive reciprocal relationship between the QoL domain values and the grouping variable (belonging to the education group) and vice versa

\begin{tabular}{l|l}
\hline Coefficients of linear discriminants & LD1 \\
\hline Physical functioning & 0.034390417 \\
\hline Role limit: emotional problems & 0.006932905 \\
\hline Role limit: physical health & 0.030501062 \\
\hline Fatigue & -0.009631083 \\
\hline Emotional wellbeing & -0.046042247 \\
\hline Social functioning & 0.001989986 \\
\hline Pain & -0.028146515 \\
\hline General health & 0.025963679
\end{tabular}

PF and emotional wellbeing (EWB) and the knowledge level on EWB. Further associations were observed between marital stage and SF, between care provision and general health $(\mathrm{GH})$, between monthly income and $\mathrm{GH}$, and between various interaction terms and the QoL domains. Because of lacking normality distributions of the QoL values within the domains (significant Shapiro-Wilk multivariate normality test results, data not shown, see also Fig. 4a-f) and low case numbers, the conclusiveness was limited. However, considering the relative robustness of the Pillai's trace regarding unbalanced data and the graphically demonstrated relationships in the scatterplots of Fig. $4 \mathrm{a}-\mathrm{f}$, these results pointed to statistical associations between the extended IC talk, the resulting knowledge level, and mid-term QoL. Other factors such as social status, PAOD stage, or ambulatory care provision are also among the determinants.

\section{Discussion}

Uninformed patients carry a higher risk of postoperative complications [9]. This study investigated patients' comprehension of the IC talk and associated factors using three questionnaires, the answers to which served as response variables. The grouping variable was the type of IC talk: standard talk or PP or WD. Covariates were age, sex, Fontaine classification, treatment group (AAA repair, inguinal TEA, or angiography with or without intervention). About $7 \%$ of those patients who fulfilled the inclusion criteria refused to take part in the study. About $74 \%$ of the included patients filled out the first questionnaire on admission, $55 \%$ the second questionnaire on the day of discharge from hospital, and $29 \%$ the third questionnaire during ambulatory follow-up (Fig. 1). The possible confounding bias resulting from this low compliance had to be considered for further analysis and conclusions. Therefore, the groups were balanced for each of the questionnaires by their own matchedpair analysis and several multivariate statistics were applied in order to confirm the results.

Only a minority of the patients of the present investigation remembered the diagnosis, the planned procedure, and possible minor and major complications. An extended IC talk had no impact on comprehension or remembrance rates compared to the standard IC talk (Fig. 2). Higher age was associated with lower knowledge within the general cohort (Table 1). This confirms the results of previous publications [10, 11]. However, other factors such as the planned treatment and self-information also affected the knowledge level of the patient. We preferred open to multiple-choice questions, providing the opportunity to answer questions in own words. In the present study, the questionnaires were predominantly related to verbal memory, which also depends on age. One can argue that recalling keywords may not ideally reflect comprehension and may even put older patients at a disadvantage. The investigators accepted keywords in any language or even abbreviations (i.e., EVAR for endovascular aortic repair) that were similar or near to what would have been a correct answer, thereby anticipating this methodical aspect. Almost all patients who were classified as not remembering any possible complication left these fields entirely empty. We conclude that adding a PowerPoint presentation or a walking diary with instructions to the standard IC talk was not enough to improve understanding of the IC talk. The best recall in some of the items was found in the aortic repair group, while the DSA and TEA group consisted of chronic patients, where repression and neglecting may be a relevant mechanism for disease coping. Meade reported that the comprehension in low-risk treatments is much higher than in high-risk treatments [12]. No differences in satisfaction or postoperative outcome could be found in the extended IC talk group compared to the standard group (Fig. 3). One reason could be the unrealistic expectations about the benefits of surgery (i.e., better walking distance after aortic aneurysm resection), as also shown in various studies [13, 14].

Belonging to the extended IC talk group was associated with a significantly higher mean score in the domain "role limitations due to physical health," and tendentially also concerning the domain "physical functioning." In order to confirm these results, a MANOVA on the QoL domain scores (Table 3) including all patients who completed the third questionnaire was calculated and revealed, among age, care provision, and income, a tendential impact of the extended IC talk and the knowledge level on various domains. The mechanism by which a onehour interactive talk may partially improve the QoL remains to be further investigated.

We hypothesize that physicians can usually not provide enough time and opportunity for just talking about the disease and answering questions. A patient may feel appreciated when the surgeon spends more time and attention on him and may develop a higher awareness for the chronicity of the disease, the underlying risk factors, realistic expectations, and the importance of lifestyle changes, confirming the 
Table 3 Results of the MANOVA on RAND36 domains including various cofactors and their interactions (indicated by the “:" sign) as explanatory terms

\begin{tabular}{|c|c|c|c|c|c|c|c|c|c|c|c|c|c|c|c|c|c|c|}
\hline \multirow[b]{2}{*}{ VARIABLE } & \multicolumn{2}{|l|}{ All } & \multicolumn{2}{|l|}{$\mathrm{PF}$} & \multicolumn{2}{|l|}{ RLEP } & \multicolumn{2}{|l|}{ RLPH } & \multicolumn{2}{|l|}{ Fat } & \multicolumn{2}{|l|}{ EWB } & \multicolumn{2}{|l|}{ SF } & \multicolumn{2}{|l|}{ Pain } & \multicolumn{2}{|l|}{ GH } \\
\hline & $\mathrm{P}$ & $p$-value & $\mathrm{F}$ & $p$-value & $\mathrm{F}$ & $p$-value & $\mathrm{F}$ & $p$-value & $\mathrm{F}$ & $p$-value & $\mathrm{F}$ & $p$-value & $\mathrm{F}$ & $p$-value & $F$ & $p$-value & $\mathrm{F}$ & $p$-value \\
\hline IC talk & 0.75 & 0.9 & 5.4 & 0.048 & 1.9 & 0.2 & 2.8 & 0.1 & 4.2 & 0.08 & 5.0 & 0.06 & 5.7 & 0.04 & 2.0 & 0.2 & 3.7 & 0.1 \\
\hline Treatment & 1.6 & 0.5 & 2.4 & 0.15 & 0.02 & 0.98 & 1.5 & 0.3 & 0.3 & 0.7 & 0.7 & 0.5 & 1.0 & 0.4 & 5.4 & 0.03 & 0.6 & 0.6 \\
\hline Fontaine & 0.9 & 0.5 & 19.2 & 0.002 & 2.0 & 0.2 & 1.1 & 0.3 & 4.5 & 0.07 & 6.5 & 0.03 & 4.9 & 0.06 & 6.5 & 0.03 & 10 & 0.01 \\
\hline Knowledge & 0.9 & 0.7 & 1.7 & 0.22 & 5.1 & 0.05 & $<0.1$ & 0.95 & 0.2 & 0.7 & 5.5 & 0.048 & 0.5 & 0.5 & 0.9 & 0.4 & 7.2 & 0.03 \\
\hline Marital st & 0.9 & 0.7 & 0.2 & 0.6 & 3.1 & 0.11 & 0.4 & 0.5 & 0.6 & 0.4 & 3.8 & 0.09 & 11.9 & 0.008 & 0.8 & 0.4 & $<0.1$ & 1.0 \\
\hline Education & 0.9 & 0.7 & 1.3 & 0.3 & 0.45 & 0.5 & 1.8 & 0.2 & 0.4 & 0.6 & 1.5 & 0.3 & 0.4 & 0.6 & 0.5 & 0.5 & 0.6 & 0.5 \\
\hline Care & 0.9 & 0.5 & 1 & 0.4 & 0.06 & 0.8 & $<0.1$ & 0.8 & 1 & 0.3 & 4.0 & 0.08 & 13.2 & 0.007 & 0.5 & 0.5 & 7.7 & 0.02 \\
\hline Income & 0.9 & 0.7 & 0.9 & 0.4 & 3.8 & 0.09 & 2.8 & 0.13 & 0.3 & 0.6 & 1.1 & 0.3 & 0.3 & 0.6 & 0.01 & 0.9 & 7.3 & 0.03 \\
\hline ICT:Treat & 0.9 & 0.6 & 0.2 & 0.7 & 0.2 & 0.7 & $<0.1$ & 0.8 & 1 & 0.3 & 0.9 & 0.4 & 0.3 & 0.6 & 0.03 & 0.9 & 3.4 & 0.1 \\
\hline ICT:Font & 1.0 & 0.4 & 0.2 & 0.9 & 0.02 & 0.9 & 0.3 & 0.6 & $<0.1$ & 0.9 & $<0.1$ & 0.8 & 0.08 & 0.8 & 2.0 & 0.2 & 5.1 & 0.05 \\
\hline Treat:Font & 0.6 & 0.9 & 0.02 & 0.9 & 3.6 & 0.09 & 0.2 & 0.7 & $<0.1$ & 0.8 & 1.2 & 0.3 & 0.2 & 0.7 & 0.07 & 0.8 & 0.02 & 0.9 \\
\hline ICT:Know & 0.9 & 0.7 & 1.4 & 0.3 & 0.8 & 0.4 & 1.7 & 0.2 & 4.5 & 0.07 & 4.3 & 0.07 & 0.7 & 0.4 & 0.03 & 0.9 & 1.1 & 0.3 \\
\hline Treat:Know & 1.8 & 0.2 & 3 & 0.1 & 0.07 & 0.9 & 0.6 & 0.6 & 0.9 & 0.4 & 0.9 & 0.5 & 2.5 & 0.1 & 0.12 & 0.9 & 0.4 & 0.7 \\
\hline Font:Know & 0.9 & 0.7 & $<0.1$ & 0.96 & $<0.1$ & 1 & $<0.1$ & 0.9 & 0.5 & 0.5 & $<0.1$ & 0.9 & 1.2 & 0.3 & 0.5 & 0.5 & 0.1 & 0.7 \\
\hline ICT:Marit & 0.9 & 0.6 & 7.6 & 0.03 & 3.3 & 0.1 & 1.6 & 0.2 & 3.2 & 0.1 & 5.8 & 0.04 & 5.4 & 0.047 & 2.6 & 0.2 & $<0.1$ & 1.0 \\
\hline Treat:Marit & 1.5 & 0.4 & 0.4 & 0.7 & 1.2 & 0.3 & 0.5 & 0.6 & 1.2 & 0.4 & 1.2 & 0.4 & 3.0 & 0.1 & 1.5 & 0.3 & 2.6 & 0.1 \\
\hline Know:Marit & 1.0 & 0.5 & 1.9 & 0.2 & 0.05 & 0.8 & 0.5 & 0.5 & 0.2 & 0.7 & 1.8 & 0.2 & 5.9 & 0.04 & 2.0 & 0.2 & 5.2 & 0.05 \\
\hline ICT:Edu & 0.3 & 1.0 & $<0.1$ & 0.95 & 0.03 & 0.9 & 0.6 & 0.4 & 0.3 & 0.6 & 0.2 & 0.7 & 0.04 & 0.8 & 0.05 & 0.8 & 0.5 & 0.5 \\
\hline Treat:Edu & 1.4 & 0.8 & 1.9 & 0.2 & 4.5 & 0.049 & 0.5 & 0.6 & 5.5 & 0.03 & 5.4 & 0.03 & 8.6 & 0.01 & 0.5 & 0.6 & 6.5 & 0.02 \\
\hline Font:Edu & 0.9 & 0.5 & 3.8 & 0.09 & 3 & 0.12 & $<0.1$ & 0.8 & 0.4 & 0.6 & 5.6 & 0.046 & 1.0 & 0.3 & 0.03 & 0.9 & 0.2 & 0.6 \\
\hline Know:Edu & 0.9 & 0.5 & 0.08 & 0.8 & 1.3 & 0.3 & $<0.1$ & 1 & 1.3 & 0.3 & 4.6 & 0.06 & 3.1 & 0.1 & 0.8 & 0.4 & 3.1 & 0.1 \\
\hline Marit:Edu & 0.8 & 0.7 & 0.2 & 0.6 & 1.5 & 0.3 & $<0.1$ & 0.8 & 0.5 & 0.5 & 2.8 & 0.1 & 10.0 & 0.01 & 0.5 & 0.5 & $<0.1$ & 1.0 \\
\hline ICT:Care & 0.7 & 0.9 & $<0.1$ & 1 & 0.6 & 0.5 & 0.1 & 0.7 & $<0.1$ & 0.9 & 0.2 & 0.7 & 3.1 & 0.1 & 0.03 & 0.9 & 0.3 & 0.6 \\
\hline Treat:Care & 0.8 & 0.8 & 5.8 & 0.04 & 0.5 & 0.5 & 0.6 & 0.5 & 0.7 & 0.4 & 0.3 & 0.6 & 0.3 & 0.6 & 0.8 & 0.4 & 3.5 & 0.1 \\
\hline Know:Care & 0.8 & 0.7 & 0.3 & 0.6 & 3.2 & 0.1 & $<0.1$ & 0.9 & 0.2 & 0.7 & 1.0 & 0.3 & 3.1 & 0.1 & 0.02 & 0.9 & 0.5 & 0.5 \\
\hline Treat:Income & 0.9 & 0.7 & 1.1 & 0.33 & 2.3 & 0.2 & 1.6 & 0.2 & 5.4 & 0.048 & 7.1 & 0.03 & 6.3 & 0.04 & 0.9 & 0.4 & 3.4 & 0.1 \\
\hline Know:Income & 0.9 & 0.7 & 4.4 & 0.06 & 0.01 & 0.9 & 2.2 & 0.2 & 0.8 & 0.4 & 1.5 & 0.3 & 0.03 & 0.9 & 1.4 & 0.3 & 0.5 & 0.5 \\
\hline Marit:Income & 0.9 & 0.6 & 1.7 & 0.22 & 1.2 & 0.3 & 0.5 & 0.5 & 3.9 & 0.08 & 8.7 & 0.02 & 0.1 & 0.7 & $<0.1$ & 1.0 & 1.2 & 0.3 \\
\hline Edu:Income & 0.9 & 0.7 & 0.13 & 0.7 & 5.5 & 0.047 & 0.3 & 0.6 & 2.7 & 0.1 & 12 & 0.008 & 3.5 & 0.1 & $<0.1$ & 1.0 & 0.6 & 0.5 \\
\hline $\begin{array}{l}\text { Know:Edu: } \\
\text { Income }\end{array}$ & 0.8 & 0.8 & 0.17 & 0.7 & $<0.1$ & 1 & 0.4 & 0.5 & 0.5 & 0.5 & $<0.1$ & 0.9 & 4.1 & 0.08 & 1.1 & 0.3 & 1.0 & 0.4 \\
\hline PF physical fu & incti & $R I$ & & . & 7 & $10 \mathrm{~m}$ & & l. & & & & & & & & & & $\begin{array}{l}\text { ing, } \\
\text { atory }\end{array}$ \\
\hline
\end{tabular}

old medical rule that talking to the patient is half the treatment. Therefore, continuous patient education should become an important part of the treatment of vascular surgery patients, including walking training groups, smoke cessation coaching, blood glucose self-management, dietary consultation, and patient support groups. However, other factors such as ambulatory care provision should also be taken into account. Within an idealized treatment network, treatment and education should be flawlessly continued in an outpatient clinic setting after discharge from hospital.

Finally, the fact that signing the IC form does not necessarily mean that the patients feel informed about the disease and all aspects of the planned treatment should lead to revisions of the legal requirements.

\section{Conclusion}

- There are several factors that should have an impact on the verbal disease knowledge level of the patient after the informed consent (IC) talk; they might be improved by additional education.

- Remembrance and comprehension rates were in general low and did not improve significantly with additional PowerPoint presentations (PP) or walking diary (WD).

- Age was inversely associated with disease knowledge. 
- Age was also inversely and WD/PP and knowledge level directly associated with mid-term quality of life (QoL).

- However, other factors, such as care provision and social status, were important as well.

- The fact that signing the IC form does not necessarily mean that the patients feel informed about the disease and all aspects of the planned treatment should lead to revisions of the legal requirements.

- Communication with and education of vascular surgery patients is one of the mainstays of treatment and should be continued beyond the legal and formal requirements during treatment and after discharge from hospital, in order to keep the QoL as high as possible.

Funding Open Access funding enabled and organized by Projekt DEAL.

\section{Compliance with ethical guidelines}

Conflict of interest A. Udelnow, V. Hecht, I. Buschmann, C. Wilbrandt, U. Barth, F. Meyer, and Z. Halloul declare that they have no competing interests. This includes, but is not limited to, any financial relationship with regard to the research presented.

Ethical standards The study was performed according to the guidelines and ethical standards of the Declaration of Helsinki for Biomedical Research from 1964 by the World Medical Association and its further amendments, the policy of the local institutional ethic committees, and according to the requirements of Good Clinical Research and Good Clinical Practice. Data generation, documentation, and evaluation was performed according to prerequisites of data protection law of the German district Saxony-Anhalt and according to federal law. With regard to the study concept, it can be stated that a (potential) danger for study participants can be definitely excluded. There is no imponderable risk or side effect for the patient as it may become possible in the use of any medication. Each patient signed an informed consent form prior to (i) surgery, including appropriate explanation of the surgical intervention and potential complications, and (ii) generation and documentation of data in the patient data registry.

Open Access This article is licensed under a Creative Commons Attribution 4.0 International License, which permits use, sharing, adaptation, distribution and reproduction in any medium or format, as long as you give appropriate credit to the original author(s) and the source, provide a link to the Creative Commons licence, and indicate if changes were made. The images or other third party material in this article are included in the article's Creative Commons licence, unless indicated otherwise in a credit line to the material. If material is not included in the article's Creative Commons licence and your intended use is not permitted by statutory regulation or exceeds the permitted use, you will need to obtain permis- sion directly from the copyright holder. To view a copy of this licence, visit http://creativecommons.org/licenses/by/4.0/.

\section{References}

1. Falagas ME, Korbila IP, Giannopoulou KP, Kondilis BK, Peppas G. Informed consent: how much and what do patients understand? Am J Surg. 2009;198(3):420-35.

2. Wann-Hansson C, WennickA. How do patients with peripheral arterial disease communicate their knowledge about their illness and treatments? A qualitative descriptive study. BMCNurs. 2016;15:29.

3. Regensteiner JG, Hiatt WR, Coll JR, Criqui MH, TreatJacobson D, McDermottMM, etal. Theimpact of peripheral arterial disease on health-related quality of life in the Peripheral Arterial Disease Awareness, Risk, and Treatment: New Resources for Survival (PARTNERS) Program. Vasc Med. 2008;13(1):15-24.

4. Hays RD, Morales LS. The RAND-36 measure of healthrelated quality oflife. Ann Med. 2001;33(5):350-7.

5. Chung WS, Lan YL, Yang MC. Psychometric testing of the short version of the world health organization quality of life (WHOQOL-BREF) questionnaire among pulmonary tuberculosis patients in Taiwan. BMC Public Health. 2012;12:630.

6. Core TR. R: a language and environment for statistical computing. 2015. https://scholar.google.de/scholar? $\mathrm{q}=$ Core $+\mathrm{TR} .+\mathrm{R}:+\mathrm{a}+$ language + and + environment + for + sta tistical+computing.+2015.\&hl=de\&as_sdt=0\&as_vis $=1 \&$ oi $=$ scholart. Accessed 11/09/2020.

7. Ho DEIK, King G, Stuart EA. Matchit: nonparametric preprocessing for parametric causal inference. J Stat Soft. 2011;42(8):28.

8. Jarek S. mvnormtest: normality test for multivariate variables. R package version 0.1-9. 2012.

9. Chen HH, Yeh ML, Yang HJ. Testing the impact of a multimedia video CD of patient-controlled analgesia on pain knowledge and pain relief in patients receiving surgery. Int J Med Inform. 2005;74(6):437-45.

10. Lavelle-Jones C, Byrne DJ, Rice P, Cuschieri A. Factors affecting quality of informed consent. BMJ. 1993;306(6882):885-90.

11. Sanwal AK, Kumar S, Sahni P, Nundy S. Informed consent in Indian patients. JR Soc Med. 1996;89(4):196-8.

12. Meade CD. Improving understanding of the informed consent process and document. Semin Oncol Nurs. 1999;15(2):124-37.

13. Lloyd A, Hayes P, Bell PR, Naylor AR. The role of risk and benefit perception in informed consent for surgery. Med Decis Making. 2001;21(2):141-9.

14. Dillon MF, Carr CJ, Feeley TM, Tierney S. Impact of the informed consent process on patients' understanding of varicose veins and their treatment. Ir J Med Sci. 2005;174(3):23-7.

Publisher's Note Springer Nature remains neutral with regard to jurisdictional claims in published maps and institutional affiliations. 